\title{
PACKING CHROMATIC NUMBER OF CERTAIN DENDRIMERS
}

\author{
S. Roy \\ Department of Mathematics \\ VIT University \\ Vellore, INDIA
}

\begin{abstract}
The packing chromatic number $\chi_{\rho}(G)$ of a graph $G$ is the smallest integer $k$ for which there exists a mapping $f: V(G) \longrightarrow\{1,2, \ldots, k\}$ such that any two vertices of color $i$ are at distance at least $i+1$. In bio-chemistry, dendrimers are synthetically produced monodisperse polymeric nanostructures with a tree-like, highly branched architecture. Applications of dendrimers typically involve conjugating other chemical species to the dendrimer surface that can function as detecting agents, affinity ligands, targeting components, radioligands, imaging agents, or pharmaceutically active compounds. In this paper, the packing chromatic number of certain dendrimers $D_{2, \mathrm{k}}, k=1,2,3,4$ are calculated. We also post some open problems.
\end{abstract}

AMS Subject Classification: $05 \mathrm{C} 15$

Key Words: packing coloring, bethe tree, dendrimer

\section{Introduction}

Let $G$ be a connected graph and $k$ be an integer, $k \geq 1$. A packing $k$-coloring of a graph $G$ is a mapping $f: V(G) \longrightarrow\{1,2, \ldots, k\}$ such that any two vertices of color $i$ are at distance at least $i+1$. The packing chromatic number $\chi_{\rho}(G)$ of $G$ is the smallest integer $k$ for which $G$ has packing $k$-coloring. The concept of packing coloring comes from the area of frequency assignment in wireless networks and was introduced by Goddard et al. [2] under the name Broad-

Received: June 16, 2017

Revised: July 25, 2017

Published: August 30, 2017 (c) 2017 Academic Publications, Ltd. url: www.acadpubl.eu 
cast coloring. It also has several applications, such as, resource placement and biological diversity. The term packing chromatic number was introduced by Bresar [1].

Dendrimers are novel synthetic polymeric systems having improved physical and chemical properties due to their unique three dimensional architecture. Dendrimers have a well defined size, shape, molecular weight and monodispersity. These are compatible with drug moieties as well as bioactive molecules like DNA, heparin and other polyanions [5]. In this paper, we calculate the packing chromatic number of certain class of dendrimers.

\section{Main Results}

Proposition 1. [2] Let $H$ be a subgraph of $G$. Then $\chi_{\rho}(H) \leq \chi_{\rho}(G)$.

Proposition 2. [2] Let $C_{n}$ be a cycle on $n$ vertices. Then $\chi_{\rho}\left(C_{n}\right)=4$ when $n$ is not a multiple of 4 .

Definition 3. [4] The Bethe tree $B_{d, k}$ is the rooted tree of $k$ levels whose root vertex has degree $d$, the vertices from level 2 to level $k-1$ have degree $d+1$ and the vertices at level $k$ have degree 1 .

Mahdieh Azari et al. [3] introduced a class of dendrimers which is constructed from copies of ordinary Bethe trees. This molecular structure can be encountered in real chemistry, e.g. in some tertiary phosphine dendrimers. Let $D_{0}$ be the graph depicted in Figure 1(a). For $d, k>0$, let $D_{d, k}$ be a series of dendrimers obtained by attaching $d$ pendent vertices to each pendent vertex of $D_{d, k-1}$ and let $D_{d, 0}=D_{0}$. The diameter of $D_{d, k}$ is $2(d+k)$.

In the following Theorems, $c(v)$ and $d(u, v)$ denote the color of a vertex $v$ and distance between vertices $u$ and $v$ respectively.

Theorem 4. For $D_{0}, \chi_{\rho}\left(D_{0}\right)=4$.

Proof. Since $C_{6}$ is a subgraph of $D_{0}$, by Propositions 1 and $2, \chi_{\rho}\left(D_{0}\right) \geq 4$. And from Figure $1(\mathrm{a}), \chi_{\rho}\left(D_{0}\right)=4$.

Theorem 5. For $D_{2,1}, \chi_{\rho}\left(D_{2,1}\right)=5$.

Proof. Suppose for the sake of contradiction that this is not true. Then $\chi_{\rho}\left(D_{2,1}\right)=4$. Label $D_{2,1}$ as it is shown in Figure 1(b). By degree sequence, the vertices of $D_{2,1}$ can be divided into three sets namely $X=\left\{a_{1}, a_{2}, v_{1}, v_{2}\right\}$, $Y=\left\{b_{1}, b_{2}, v_{3}, v_{4}\right\}$ and $Z=\left\{c_{1}, c_{2}, v_{5}, v_{6}\right\}$ and by assumption atmost one 


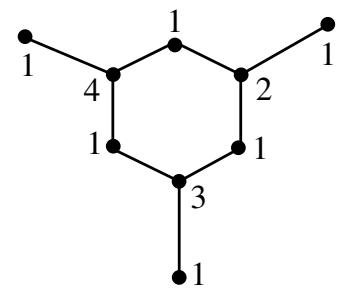

(a)

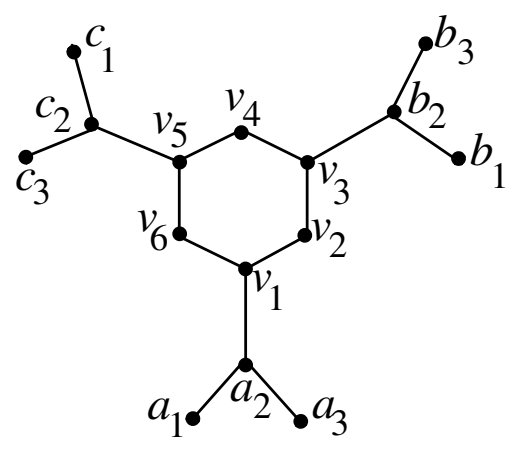

(b)

Figure 1: (a) Packing coloring of $D_{0}$. (b) Labelling of $D_{2,1}$

vertex of these sets should receive color 4 . Considering the set $X$ for the discussion, it holds true for other vertices in the sets $Y$ and $Z$ due to their degree sequence. Our aim is to minimize the packing coloring. Therefore, we try to label maximum vertices with minimum colors.

Case I: when $c\left(a_{1}\right)=4$, let $c\left(a_{2}\right)=1, c\left(v_{1}\right)=3$ and $c\left(a_{3}\right)=2$. Since $d\left(a_{2},\left\{v_{2}, v_{6}\right\}\right)=2$ and $d\left(v_{2}, v_{6}\right)=2, c\left(v_{2}\right)=c\left(v_{6}\right)=1$. Since $d\left(a_{3}, v_{3}\right)=4$, $c\left(v_{3}\right)=2$. Now for vertex $v_{5}$, the only hope is to get a color greater than 4 .

Case II: when $c\left(a_{2}\right)=4$, let $c\left(a_{1}\right)=c\left(v_{1}\right)=c\left(a_{3}\right)=1$. Since $d\left(v_{1},\left\{v_{3}, v_{5}\right\}\right)=$ 2 and $\left.d\left(v_{3}, v_{5}\right\}\right)=2, c\left(v_{3}\right)=c\left(v_{5}\right)=1$. Now, vertices $v_{2}$ and $v_{6}$ can be colored 2 and 3 respectively. Since vertex $v_{3}$ is colored $1, d\left(v_{4},\left\{v_{2}, v_{6}\right\}\right)=2$ and $d\left(a_{2}, v_{4}\right)=4$, the vertex $v_{3}$ should receive a color greater than 4 .

Case III: when $c\left(v_{1}\right)=4$, let $c\left(v_{1}\right)=c\left(v_{2}\right)=c\left(a_{2}\right)=1$. Since $d\left(v_{2},\left\{v_{4}, b_{2}\right\}\right)=$ 2 and $d\left(v_{4}, b_{2}\right)=2, c\left(v_{4}\right)=c\left(b_{2}\right)=1$. Now, vertices $a_{1}$ and $a_{3}$ can only be colored with 2 and 3 respectively. Since $d\left(a_{3},\left\{v_{5}, b_{3}\right\}\right) \geq 4$ and $d\left(v_{5}, b_{3}\right)=4$, $c\left(v_{5}\right)=c\left(b_{3}\right)=3$. Now for vertex $b_{1}$, the only possibility is to get a color greater than 4 .

Case IV: when $c\left(v_{2}\right)=4$, let $c\left(v_{1}\right)=1, c\left(v_{3}\right)=1$ and $c\left(a_{2}\right)=2$. Since vertices $a_{1}, a_{3}, b_{1}, b_{3}$ and $v_{5}$ are at distance $\geq 2$ to each other, $c\left(a_{1}\right)=c\left(a_{3}\right)$ $=c\left(b_{1}\right)=c\left(b_{3}\right)=c\left(v_{5}\right)=1$. Now the vertex $v_{6}$ can receive color 2 or 3 but let $c\left(v_{6}\right)=3$. Since $d\left(v_{6}, b_{2}\right)=4$ and $d\left(a_{2}, v_{4}\right) \geq 3, c\left(b_{2}\right)=3$ and $c\left(v_{4}\right)=2$. For the vertex $c_{2}$, the vertices received colors $2,3,4$ are at distance $\leq 2$, at distance $\leq 3$, at distance $\leq 4$ respectively and $c\left(v_{5}\right)=1$. Thus, the vertex $c_{2}$ should receive a color greater than 4 . 


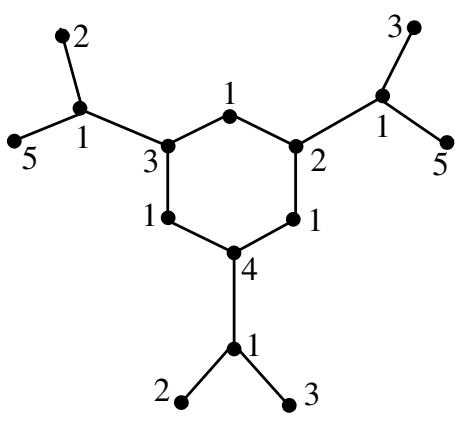

(a)

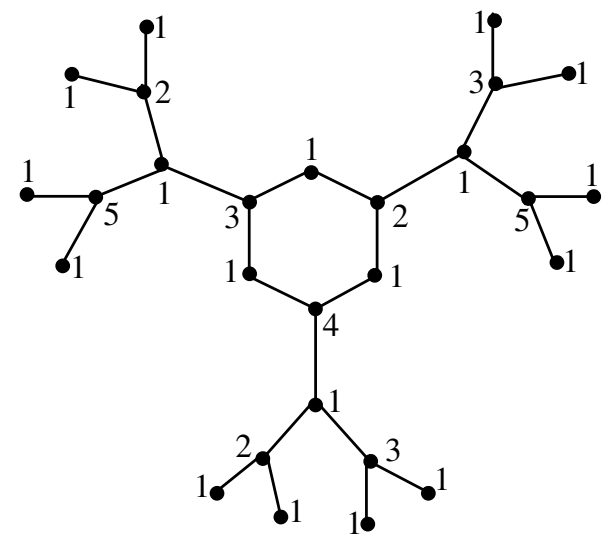

(b)

Figure 2: (a) Packing coloring of $D_{2,1}$. (b) Packing coloring of $D_{2,2}$

Hence from above four Cases, $\chi_{\rho}\left(D_{2,1}\right) \geq 5$. From Figure 2(a), we conclude that $\chi_{\rho}\left(D_{2,1}\right)=5$.

Theorem 6. For $D_{2,2}, \chi_{\rho}\left(D_{2,2}\right)=5$.

Proof. Since $D_{2,1}$ is a subgraph of $D_{2,2}$, by Propositions 1 and $2, \chi_{\rho}\left(D_{2,2}\right) \geq$ 5. And from Figure 2(b), $\chi_{\rho}\left(D_{2,2}\right)=5$.

Theorem 7. For $D_{2,3}$ and $D_{2,4}, 5 \leq \chi_{\rho}\left(D_{2,3}\right), \chi_{\rho}\left(D_{2,4}\right) \leq 6$.

Proof. Since $D_{2,2}$ is a subgraph of $D_{2,3}$ and $D_{2,4}$, by Propositions 1 and 2 , $\chi_{\rho}\left(D_{2,3}\right) \geq 5$ and $\chi_{\rho}\left(D_{2,4}\right) \geq 5$. And from Figures $3(\mathrm{a})$ and $3(\mathrm{~b}), \chi_{\rho}\left(D_{2,3}\right) \leq 6$ and $\chi_{\rho}\left(D_{2,4}\right) \leq 6$.

\section{Open Problems}

Problem 1. Determining $\chi_{\rho}\left(D_{2, k}\right)$ for $k>4$ is challenging and the problem remains open.

Problem 2. $\chi_{\rho}\left(D_{2, k}\right) \leq 2(d+k)$. 


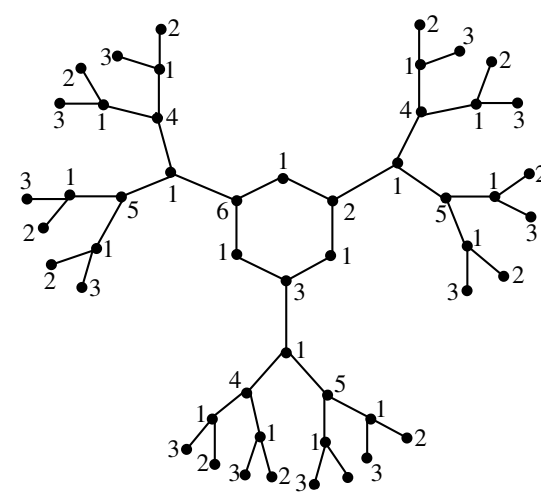

(a)

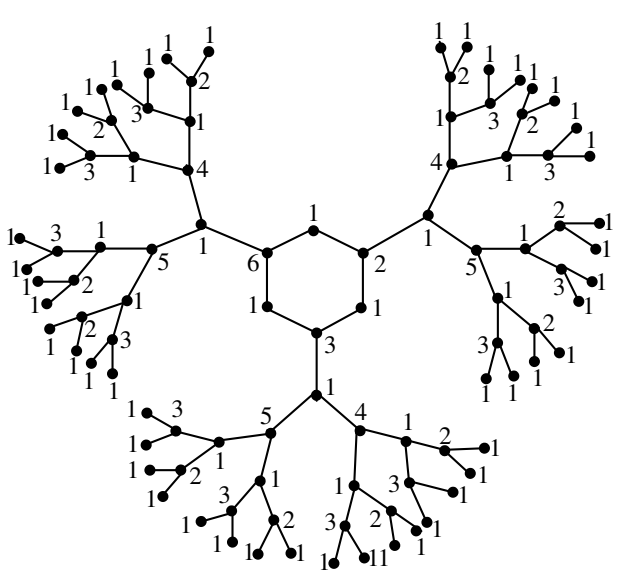

(b)

Figure 3: (a) Packing coloring of $D_{2,3}$ (b) Packing coloring of $D_{2,4}$

\section{Conclusion}

In this paper the packing chromatic number of $D_{2, k}$ for $k=1,2,3,4$ is calculated and the problem of packing coloring for other chemical structures is under investigation.

\section{References}

[1] B. Brešar, S. Klavžar, D. F. Rall, On the Packing Chromatic Number of Cartesian Products, Hexagonal Lattice and Trees, Discrete Appl. Math., 155(17) (2007), 23032311, doi: 10.1016/j.dam.2007.06.008.

[2] W. Goddard, S. M. Hedetniemi, S. T. Hedetniemi, J. M. Harris, D. F. Rall, Broadcast Chromatic Numbers of Graphs, Ars Combin., 86 (2008), 33-49.

[3] Mahdieh Azari, Ali Iranmanesh, Mircea V. Diudea, Vertex-Eccentricity Descriptors in Dendrimers, Studia Ubb Chemia., LXII(1) (2017), 129-142, doi: 10.24193/subbchem.2017.1.11.

[4] María Robbiano, I. Gutman, R. Jiménez, B. San Martín, Spectra of Copies of Bethe Trees Attached to Path and Applications, Bulletin Classe Des Sciences Mathematiques Et Natturalles., 137(33) (2008), 59-81.

[5] Surendra Tripathy, Malay K Das, Dendrimers and their Applications as Novel Drug Delivery Carriers, Journal of Applied Pharmaceutical Science., 3(09) (2013), 142-149, doi: $10.7324 / J A P S .2013 .3924$. 
